\title{
Prueba de caminata de carga progresiva (Incremental Shuttle Walking Test) en niños sanos
}

\author{
DANIEL CIUDAD A.*, PATRICIA DÍAZ P.*, JOSÉ ORELLANA U.* y CATALINA SOTO M.*
}

\section{Incremental shuttle walking test: In healthy children}

Background: The "incremental shuttle walking test" (ISWT) is an incremental test that allows the functional evaluation of respiratory diseases. It shows a greater correlation between distance walked and the maximum $\mathrm{O}_{2}$ uptake compared to the six-minute walking test. Presently we do not have reference values in Chilean pediatric population. Objective: To know the distance walked by children in the test. Material and Method: This was a correlational descriptive study. The final sample consisted of 60 subjects (35 boys and 25 girls) randomly selected from an age range from 10 to 14 years-old. During the test, walked distance (WD) in meters, basal and final heart rate (HR), dyspnea, subjective sensation of fatigue (SSF), gender, weight $(\mathrm{kg})$, height $(\mathrm{m})$ and cardiac response to exercise (CRE) were recorded. Descriptive statistics were performed in both series followed by a t-test to look for differences among genders. Pearson's correlation coefficient was used to verify the correlation between variables. Results were expressed as mean $\pm S D$. Results: Mean of WD in all the 60 children together was 596.2 $\pm 178.7 \mathrm{~m}$; in average WD in girls series was $531.6 \pm 136.5 \mathrm{~m}$ and in boys series was $642.3 \pm 192.4 \mathrm{~m}$ $(p=0.017)$. The average height for boys was $1.50 \pm 0.08 \mathrm{~m}$ and for girls was $1.53 \pm 0.08 \mathrm{~m}(p=0.245)$. The average BMI for boys was $18.3 \pm 1.6 \mathrm{~kg} / \mathrm{m}^{2}$ and for girls was $19.3 \pm 2.2 \mathrm{~kg} / \mathrm{m}^{2}(p=0.041)$. Pearson's correlation coefficient between the average of meters walked for all the 60 children together and CRE was $0.570(p<0.001)$. Conclusions: Boys showed a greater WD than girls in ISWT. There was no influence of age, weight, height or BMI on distance walked on the ISWT. A positive correlation is shown between the WD and the CRE.

Key words: Child; dyspnea; walking; exercise; heart rate.

\section{Resumen}

Introducción: La prueba de caminata de carga progresiva (PCCP) es una prueba incremental que permite la evaluación funcional de las enfermedades respiratorias. Esta prueba muestra una mayor correlación entre la distancia recorrida y el consumo máximo de $\mathrm{O}_{2}$ comparada con la prueba de marcha de seis minutos. En la actualidad, no contamos con valores de referencia en la población pediátrica nacional. Objetivo: Conocer la distancia recorrida en niños en la prueba. Material y Método: Estudio descriptivo correlacional. La muestra final fue de 60 sujetos (35 niños y 25 niñas) elegidos al azar en un rango de edad de 10 a 14 años. Durante la prueba se registró la distancia recorrida (DR) en metros, frecuencia cardíaca basal y final (FC), disnea, sensación subjetiva de fatiga (SSF), género, peso $(\mathrm{kg})$, talla (m) y respuesta cardíaca al ejercicio (RCE). Se realizó estadística descriptiva en ambos grupos seguida de una prueba " $t$ " para analizar las diferencias entre género. Se aplicó el coeficiente de correlación de Pearson para verificar la correlación entre las variables. Los resultados fueron expresados en medias \pm desviación estándar (DE). Resultados: El promedio de la DR del conjunto de los 60 menores fue 596,2 $\pm 178,7 \mathrm{~m}$; para la DR en las niñas el promedio fue de 531,6 $\pm 136,4 \mathrm{~m}$ y la DR promedio en los niños de 642,3 $\pm 192,4 \mathrm{~m}(\mathrm{p}=0,017)$. El promedio de la talla para los niños fue de

Financiado por proyecto UVA 1315. Universidad de Valparaíso.

* Escuela de Kinesiología, Facultad de Medicina, Universidad de Valparaíso. 
1,50 $\pm 0,08 m$ y para las niñas de 1,53 $\pm 0,08 \mathrm{~m}$. El coeficiente de correlación de Pearson fue de 0,570 $(p<0,001)$ entre el promedio de DR para ambos géneros y la RCE. Conclusiones: Los niños alcanzan una mayor DR que las niñas en la PCCP. No se encontró influencia de la edad, peso, estatura o IMC en la distancia recorrida para la PCCP. En el conjunto de los 60 niños se observó una correlación positiva entre la DR y la RCE.

Palabras clave: Niños; disnea; caminata; ejercicio; frecuencia cardiaca.

\section{Introducción}

La prueba de caminata de carga progresiva (PCCP), también conocida como incremental shuttle walking test (ISWT), es una prueba incremental sub-máxima de ejercicio, estandarizada, sencilla, económica y altamente reproducible. Posee buena correlación con la calidad de vida de los sujetos. Cumple un papel importante en la evaluación funcional de las enfermedades respiratorias, además determina el efecto de una intervención en individuos con estas afecciones. Esta prueba se realiza en un pasillo plano de 10 metros, señalizados por dos conos y es dirigida por estímulos sonoros, los cuales indican el momento en que se incrementa la velocidad (tres señales acústicas) y el cambio de sentido en el extremo del circuito (una señal acústica). La PCCP consta de 12 niveles, un minuto por cada nivel. La prueba finaliza cuando el niño indica que no es capaz de continuar; o es incapaz de mantener la velocidad para alcanzar a llegar al cono correspondiente antes de la señal acústica dos veces consecutivas ${ }^{1}$.

La progresiva utilización de la PCCP para el estudio de niños con enfermedades respiratoria ha puesto de manifiesto la falta de valores de referencia para su valoración clínica. Considerando la problemática señalada Probst y cols. en el año 2012 y Lanza y cols. en el año 2015, propusieron ecuaciones de predicción para niños y adolescentes en Brasil2,3.

En la actualidad, a nivel nacional, no se cuenta con valores de referencia en menores de edad, lo que dificulta su correcta interpretación

El objetivo del estudio fue describir la distancia a recorrer por niños sanos en la PCCP, conocer si se presentan diferencias entre género y determinar las posibles relaciones entre variables antropométricas y la distancia recorrida en la prueba.

\section{Material y Método}

Estudio descriptivo de tipo transversal correlacional, donde se evalúa la distancia recorrida en una PCCP. Para la determinación de la muestra en estudio, se consideró la población total de estudiantes chilenos, en la ciudad de Valparaíso, entre 6 a 14 años pertenecientes a establecimientos educacionales de la Corporación Municipal de Valparaíso (CORMUVAL) el año 2014, siendo ésta igual a 22.140 estudiantes. Considerando un $20 \%$ de deserción en la población, más referencias bibliográficas ${ }^{2,3,4}$, se determinó una muestra final de 144 alumnos.

De los 56 establecimientos educacionales pertenecientes a la CORMUVAL, se escogieron aleatoriamente 5. Con la muestra determinada de 144 participantes, y los 5 establecimientos definidos, se procedió a seleccionar el número de alumnos a participar para cada establecimiento, según el número de matrículas oficial al año 2016.

El total de matrículas de los cinco establecimientos entre primero básico a primero medio fue de 1.310 alumnos. Para determinar el número de niños por nivel se obtiene el cociente de la muestra total dividido por el número de cursos (144/9) resultando 16 niños por nivel. Finalmente, para determinar el número de alumnos por curso de cada establecimiento, se realizó una proporción con las siguientes variables: total de alumnos por curso, población total de alumnos de los cinco establecimientos y número total de la muestra.

De los 144 alumnos escogidos de manera aleatoria definidos para participar en el estudio, se incorporaron 131 sujetos, entre 6 a 14 años. De los incorporados, 119 sujetos (68 niños y 51 niñas) cumplían los criterios de inclusión del estudio: Rango de edad entre 6 a 14 años, índice de masa corporal (IMC) de rango normal a sobre peso, sin antecedentes de hospitalización en los tres últimos meses, sin historia de tabaquismo activo, sin consumo de medicamentos en forma permanente ni sintomatología respiratoria. Consentimiento informado para padres y asentimiento informado para participantes firmados, y que según el cuestionario de salud KidScreen $52^{5}$ aplicado, correspondieran a niños sanos.

En el presente estudio, se determinó considerar una muestra final de niños, entre 10 a 14 años, debido a la existencia de mayor equilibrio en 
cantidad de alumnos por edad para ambos sexos y un índice de masa corporal (IMC) en rango de normalidad para cada edad y sexo, quedando así un total de 60 sujetos ( 35 niños y 25 niñas).

Durante la ejecución de la prueba se registró la DR en metros, FC basal y final medida en latidos por minutos (lpm), SSF medida a través de la escala de Borg, disnea a través de la escala de Borg modificada $^{6}$, sexo, peso $(\mathrm{kg})$, talla $(\mathrm{m})$ y mediante una fórmula de acuerdo a las variables ya registradas, se determinó la respuesta cardíaca al ejercicio $(\mathrm{RCE})^{7}$ definida como: $\% \mathrm{FC}$ reserva $=\mathrm{FC}$ final- FC reposo/FC máx. Teórica-FC reposo.

La realización del estudio fue aprobado por la Corporación Municipal de Valparaíso para el Desarrollo Social. Cada participante debió firmar un asentimiento informado y a los padres o tutores se les entregó consentimiento informado para su aprobación. Este estudio fue aprobado por el Comité de Bioética de la Facultad de Medicina de la Universidad de Valparaíso ( $\left.\mathrm{N}^{\mathrm{o}} 41 / 2015\right)$.

\section{Análisis estadístico}

Mediante el software SPSS 22.0 se realizó estadística descriptiva en ambos grupos. Se aplicó la prueba de Kolmogorov-Smirnov para confirmar la normalidad de distribución de los datos, y t Student para muestras independientes con el fin de verificar las diferencias entre ambos sexos. A su vez, para establecer el grado de correlación entre los metros recorridos con las distintas variables evaluadas se utilizó la prueba de correlación de Pearson. Los resultados son presentados como promedios \pm DS.

\section{Resultados}

El promedio de la distancia recorrida de la muestra fue de 596,2 $\pm 178,7 \mathrm{~m}$. Para la DR en mujeres, la media fue de $531,6 \pm 136,5 \mathrm{~m}$ y la DR en hombres tuvo una media $642,3 \pm 192,4$ metros $(\mathrm{p}=0,017)$. El promedio de la talla para los hombres fue de $1,50 \pm 0,08$ metros y para las mujeres $1,53 \pm 0,08$ metros $(p=0,245)$. El coeficiente de correlación de Pearson fue de 0,570 $(p<0,001)$ entre el promedio de metros recorridos para ambos géneros y la respuesta cardiaca al ejercicio (Figura 1). No hubo influencia de la edad $(r=0,224, p=0,085)$, peso $(r=0,170$, $\mathrm{p}=0,194)$, estatura $(\mathrm{r}=0,197, \mathrm{p}=0,131)$ o IMC $(\mathrm{r}=0,062, \mathrm{p}=0,638)$ en la distancia recorrida para la PCCP en la muestra de estudio.

En cuanto al promedio de los metros recorridos, los niños caminaron $110,7 \mathrm{~m}(\mathrm{p}=0,017)$ metros más que las niñas.

De las variables estudiadas, la DR, y el IMC presenta diferencia estadísticamente significativa entre hombres y mujeres.

\section{Discusión}

La distancia a recorrer por niños entre 10 a 14 años es de 596,2 $\mathrm{m}$ valor muy por debajo de los 981,4 m reportados por Lanza FC y cols ${ }^{2}$. Esta diferencia de $385,2 \mathrm{~m}$. se explica porque en el estudio brasileño a los participantes se les permitió correr, sumado a que el protocolo utilizado contaba con 3 niveles adicionales de velocidad

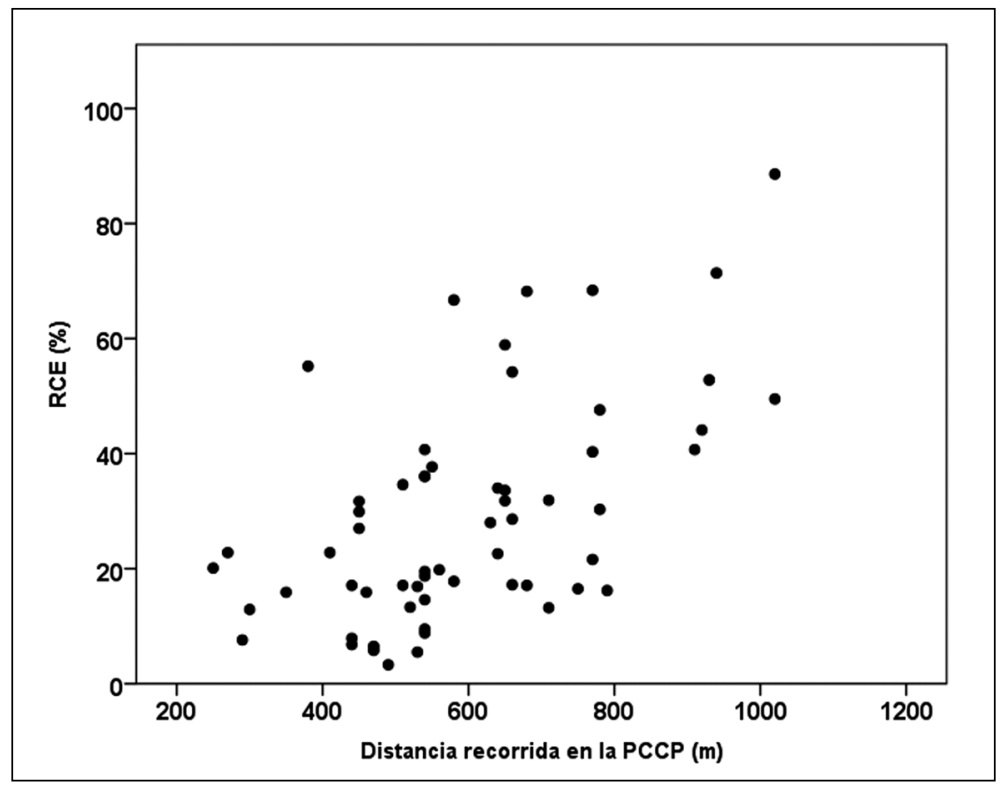

Figura 1. Dispersión de la distancia recorrida en la prueba de caminata de carga progresiva (PCCP) y la respuesta cardíaca al ejercicio (RCE) en los 60 niños evaluados. Coeficiente de correlación de Pearson: 0,57 ( $\mathrm{p}<0,001)$. 
Tabla 1. Características antropométricas, distancia recorrida, frecuencia cardíaca y respuesta cardíaca al ejercicio en los 60 niños estudiados

\begin{tabular}{|lcccc|}
\hline & $\begin{array}{c}\text { Total grupo } \\
(\mathbf{n = 6 0 )}\end{array}$ & $\begin{array}{c}\text { Hombres } \\
(\mathbf{n = 3 5 )}\end{array}$ & $\begin{array}{c}\text { Mujeres } \\
(\mathbf{n = 2 5})\end{array}$ & Valor de $\mathbf{p}^{*}$ \\
\hline Distancia (m) & $596,2 \pm 178,7$ & $642,3 \pm 192,4$ & $531,6 \pm 136,5$ & 0,017 \\
\hline Edad (años) & $12,2 \pm 1,4$ & $12,2 \pm 1,4$ & $12,7 \pm 1,4$ & 0,168 \\
\hline Peso (kg) & $46,1 \pm 11,2$ & $41,9 \pm 7,0$ & $45,5 \pm 6,9$ & 0,052 \\
\hline Estatura (m) & $1,51 \pm 0,09$ & $1,50 \pm 0,08$ & $1,53 \pm 0,08$ & 0,245 \\
\hline IMC (kg/m $\left.{ }^{2}\right)$ & $19,8 \pm 3,0$ & $18,3 \pm 1,6$ & $19,3 \pm 2,2$ & 0,041 \\
\hline Frecuencia cardiaca reposo (lpm) & $84,3 \pm 18,9$ & $83,1 \pm 19,7$ & $89,4 \pm 19,0$ & 0,224 \\
\hline Frecuencia cardiaca final (lpm) & $119,1 \pm 27,8$ & $118,1 \pm 28,9$ & $125,2 \pm 24,5$ & 0,329 \\
\hline Respuesta cardiaca al ejercicio (\%) & $28,5 \pm 19,2$ & $28,7 \pm 19,8$ & $29,8 \pm 18,7$ & 0,835 \\
\hline
\end{tabular}

Valores expresados como media \pm desviación estándar. *Según t-test de Student para muestra independientes.

(15 niveles), adaptaciones también presentes en otras investigaciones ${ }^{2,3,8,9}$. Se comprueba una correlación positiva entre la DR y la RCE en nuestro estudio, sin embargo la RCE no supera el $40 \%$ (Tabla 1) de un esfuerzo máximo, situando a la prueba en un nivel de intensidad leve ${ }^{7}$. Lo anterior nos hace pensar si realmente la PCCP representa un esfuerzo máximo para la población infantil, sobre todo si pretendemos evaluar la respuesta cardiopulmonar frente al ejercicio, que en suma representa la capacidad del cuerpo humano de poder capturar, transportar y utilizar el oxígeno durante una actividad física ${ }^{12}$. Intensidades de trabajo físico cercanas al $85 \%$ de una frecuencia cardiaca de reserva representan mejor el componente aeróbico ${ }^{10}$, lo que podría lograse haciendo los ajustes ya observados en esta prueba o bien seleccionando otra.

Al igual que en otras pruebas de marcha la DR de los hombres es mayor que el de las mujeres $^{13-15}$. Los $110 \mathrm{~m}$ adicionales recorridos por los hombres $(642,3 \pm 192,4$ versus $531,6 \pm 136,5 \mathrm{~m})$ puede explicarse por las diferencias en el desarrollo y crecimiento de los niños. Durante la realización de actividades físicas en niños, suceden diversos cambios y adaptaciones fisiológicas, que varían según sexo, edad y estado nutricional. $\mathrm{Si}$ bien es cierto en ambos géneros aumenta considerablemente la capacidad aeróbica, la práctica deportiva regular en la etapa prepuberal ocasiona un aumento de la actividad enzimática oxidativa, fundamentalmente de las enzimas succinato deshidrogenasa, citocromo oxidasa y palmitil CoA sintetasa. El aumento de las reservas de glucógeno muscular, favorecen la posibilidad de conseguir un mayor rendimiento en actividades realizadas en régimen aeróbico. Sin embargo, un elemento diferenciador entre niñas y niños es la presencia de la testosterona. Esta hormona, que se constituye como un elemento gravitante en el rendimiento deportivo, responsable del anabolismo muscular y que condiciona en gran medida el nivel de fuerza que puede desarrollar una persona, aparece en concentraciones apreciables en la sangre a partir de la pubertad. Así, hacia la edad aproximada de 11 años, se hallan concentraciones de $60 \mathrm{mg} / 100 \mathrm{~mL}$ de sangre en ambos sexos. Terminada la pubertad, aproximadamente a la edad de 15 años, ya se detectan concentraciones de hasta $600 \mathrm{mg} / 100 \mathrm{~mL}$ de sangre en el varón y $85 \mathrm{mg} / 100 \mathrm{~mL}$ de sangre en la mujer. Esto trae como consecuencia aumento de una de las características físicas de mayor relevancia entre hombre y mujeres: desarrollo de la fuerza y por ende de su potencia muscular y resistencia. A partir del aumento de la fuerza y la resistencia, el varón logra diferencias importantes en el desempeño y resultado en la mayoría de las actividades deportivas $^{10,11}$.

\section{Limitaciones del estudio}

La PCCP se realizó según el protocolo original propuesto por Singh y cols ${ }^{1}$. en 1992 . Sin embargo, la disponibilidad de los centros educacionales y organización de la clase de educación física obligó al equipo de investigadores a realizar solo una prueba.

\section{Agradecimientos}

Los autores agradecen a la directora del área de Educación de la Corporación Municipal de Valparaíso, Sra. Cecilia Pinochet por autorizar el 
ingreso a los establecimientos educacionales seleccionados y al proyecto UVA 1315 "Los Estudiantes Primero" de la Universidad de Valparaíso por la confianza y el financiamiento otorgado.

\section{Bibliografía}

1.- SINGH SJ, MORGAN MDL, SCOTT S, WALTERS D, HARDMAN AE. Development of a shuttle walking test of disability in patients with chronic airflow obstruction. Thorax 1992; 47: 1019-24.

2.- PROBST VS, HERNANDES NA, TEIXEIRA DC, FELCAR JM, MESQUITA RB, GONÇALVES CG, et al Reference values for the incremental shuttle walking test. Respir Med 2012; 106: 243-8.

3.- LANZA FC, ZAGATTO PE, CUNHA SJ, RODRIGUES SJP, GONCALVES ITV, MILANI Z DJ, et al. Reference Equation for the Incremental Shuttle Walk Test in Children and Adolescents. Journal of Pediatrics 2015; 1057-61.

4.- SELVADURAI HC, COOPER CP, MEYERS M. Validation of Shuttle tests in Children with Cystic Fiabrosis. Pediatric Pulmonary 2003; 35: 133-8.

5.- KIDSCREEN-52. Instrumento de medida de la Calidad de Vida para Niños y Adolescentes. Disponible en: https://www.kidscreen.org/espa $\% \mathrm{C} 3 \% \mathrm{~B} 1$ ol/cuestionario-kidscreen/kidscreen-52/ [Consultado el 6 de octubre de 2017].

6.- BORG G. Psychophysical bases of perceived exertion. J Med Sci Sports Exercise 1982; 14: 377-81.

7.- KARVONEN MJ, KENTALA E, MUSTALA O. The effects of training on heart rate; a longitudinal study.
Annales Medicinae Experimentalis et Biologiae Fenniae 1957, 35: 307-15.

8.- GOLIAS C, MESQUITA R, HAYASHI D, MERLI MF. Does the incremental dhuttle walking test requiere maximal effort in healthy subjects of diffent ages? Physiotherapy 2015; 141-6.

9.- JÜRGENSEN SP, ANTUNES LC, TANNI SE, BANOV MC, LUCHETA PA, BUCCERONI AF, et al The incremental Shuttle Walking test in older Brazilian adults. Respiration 2011; 81: 223-8.

10.- WILMORE J, COSTILL D. Fisiología del Esfuerzo y del Deporte. 5a Edición. Barcelona, España: editorial Paidotribo. 2004.

11.- LÓPEZ JL, FERNÁNDEZ A. Fisiología del Ejercicio. $3^{\mathrm{a}}$ edición. Madrid, España: Editorial Panamericana. 2006.

12.- VILARÓ J. Prueba de marcha de 6 minutos En: Burgos P. Casan. Procedimientos de evaluación de la función pulmonar II. F. Capítulo 3.2 Manual SEPAR de procedimientos 2004; págs: 100-13.

13.- Tablas de IMC y tablas de IMC para la edad, de niños(as) y adolescentes de 5 a 18 años de edad y tablas de IMC para adultos(as) no embarazadas, no lactantes $\geq 19$ años de edad. Disponible en: https://www.fantaproject.org [Consultado el 6 de octubre de 2017].

14.- ATS Statement. Guidelines for the Six-Minute Walk Test. American Thoracic Society. American Journal of Respiratory and Critical Care Medicine 2002; 166: 1117.

15.- BURGOS RINCÓN F, CASAN CLARÁ P, ORTEGA RUIZ F, PUENTE-MAESTU L, TOGORES SOLIVELLAS B. Normativa SEPAR. Pruebas de ejercicio cardiopulmonar. Arch Bronconeumol 2001; 37: 247-68.

Correspondencia a:

Daniel Ciudad Antognini, PhD

Escuela de Kinesiología.

Universidad de Valparaíso.

Facultad de Medicina.

Angamos 655 Reñaca-Viña del Mar.

Email: daniel.ciudad@uv.cl 